\title{
EVALUATION OF THE POLYMERASE CHAIN REACTION ANALYSIS FOR DIAGNOSIS OF FALCIPARUM MALARIA IN DELHI, INDIA
}

\author{
*S Nandwani, M Mathur, S Rawat
}

\begin{abstract}
Plasmodium falciparum infections are frequently fatal if untreated and hence need to be diagnosed and treated early. Malaria diagnosis, with conventional Giemsa staining as a gold standard, has had several limitations. New rapid and accurate methods are needed for diagnosis. In this study, polymerase chain reaction (PCR) analysis specific for diagnosis of $P$. falciparum was evaluated. For the study, blood samples were collected from 310 patients suspected of having malaria. PCR analysis for P. falciparum from venous blood and at the same time Giemsa staining of thick and thin blood smears was done. A total of $160(51.6 \%)$ samples were positive for malarial parasite of which $63(39.4 \%)$ were positive for $P$. falciparum by Giemsa staining while 61 (38.1\%) were positive for P. falciparum by PCR analysis. Giemsa staining was time consuming, laborious and may give poor results in cases with low parasitaemia. The PCR analysis for P. falciparum was able to detect 3 cases of low parasitaemia missed initially on Giemsa staining, was $96.8 \%$ sensitive, 100\% specific but was very costly, needed a lot of practice and standardization and was time consuming. PCR analysis can be used to supplement the conventional Giemsa staining for reliable diagnosis of falciparum malaria especially in cases with low parasitaemia.
\end{abstract}

Key words: Malaria, P. falciparum, diagnosis, polymerase chain reaction.

Of all the human malarial parasites Plasmodium falciparum is the most pathogenic and is frequently fatal if untreated. In India a total of 1.82 million cases of malaria and 0.89 million $P$. falciparum cases with 902 deaths have been reported during 2002. ${ }^{1}$ Malaria diagnosis has had several limitations. The conventional Giemsa staining of peripheral blood smears remains the gold standard for diagnosis of malaria in developing endemic countries. ${ }^{2}$ However, this technique is time consuming, requires training and may give poor results in cases with low parasitaemia. ${ }^{3}$ In addition, in patients with $P$. falciparum malaria, sometimes the parasites can be sequestered and are not present in peripheral blood. Thus a $P$. falciparum infection could be missed due to absence of the parasite in a blood film. ${ }^{4}$ In view of the above, a study was done to compare microscopic examination of blood film with polymerase chain reaction (PCR) analysis specific for $P$. falciparum diagnosis.

\section{Materials and Methods}

A total of 310 blood samples were collected from patients clinically suspected of malaria coming to the malaria centre at a dispensary in Nand Nagri, Delhi and OPD Guru Teg Bahadur Hospital, Shahdara, Delhi. A proforma including relevant details of history was filled for each patient.

*Corresponding author (email: <sumisahjwani@yahoo.com>)

Department of Microbiology, University College of Medical Sciences and Guru Teg Bahadur Hospital, Shahdara, New Delhi - 110 095, India.

Received: 20-09-2004

Accepted: 30-10-2004
Venous blood samples were collected in an EDTA vial from all the patients and stored at $4{ }^{\circ} \mathrm{C}$ for PCR analysis. At the same time thick and thin blood smears were prepared. Giemsa staining of blood smears was done and examined under a standard light microscope. One hundred microscopic thick field films under an oil immersion objective at 1000x magnification were seen for any malarial parasite. Any sample negative by Giemsa thick film but positive by PCR analysis for malaria was re-examined by Giemsa thick film (up to 600 fields). The final result was taken as the gold standard. Thin films were screened for species identification. Parasitaemia was counted by counting the number of parasites per 200 leucocytes and multiplying it by 40 to give parasites per microlitre of blood. ${ }^{5}$ The PCR analysis for P. falciparum was done for all the samples. DNA extraction and purification from EDTA blood samples was done using the WIZARD Genomic DNA purification kit from PROMEGA Corp. USA. The extracted DNA was thereafter amplified by the PCR using a set of primers belonging to MSP 1 block 17 as this region has been found to be highly conserved in P. falciparum. ${ }^{6}$ Each primer was used at an individual concentration of $250 \mathrm{nM}$. MSP 1 block 17 consists of the following primer pair:

\section{Primer 1-5' CA T TTC ALA ACA CCA ATG CGT 3'}

\section{Primer 2-5' TCC TAA GAA GTT AGA GGA ACT GCA G 3'}

A reaction cocktail was prepared with $1 \mu \mathrm{L}$ of each primer, $2 \mu \mathrm{L}$ of dNTPs ( $125 \mu \mathrm{M}$ each), $5 \mu \mathrm{L}$ of test DNA template, 1 $\mu \mathrm{L}$ of Taq Polymerase, $5 \mu \mathrm{L}$ of $10 \mathrm{x}$ buffer and $35 \mu \mathrm{L}$ distilled water to make a total of $50 \mu \mathrm{L}$ volume. The mixture was overlayed with $50 \mu \mathrm{L}$ of mineral oil to prevent evaporation of sample and loaded in the thermocycler. The thermocycler 
was programmed as follows. Hot start $-95^{\circ} \mathrm{C}$ for $5 \mathrm{~min}$; initial denaturation- $94{ }^{\circ} \mathrm{C}$ for $1 \mathrm{~min}$; annealing $-55^{\circ} \mathrm{C}$ for $2 \mathrm{~min}$; extension $-72{ }^{\circ} \mathrm{C}$ for $2 \mathrm{~min}$; denaturation $-94^{\circ} \mathrm{C}$ for $1 \mathrm{~min}$. A total of 39 cycles were used and final annealing and extension was for $5 \mathrm{~min}$. after which the reaction was stopped and the amplified product was detected by electrophoresis on $2 \%$ agarose gel containing ethidium bromide and visualized on an ultraviolet transilluminator. Gel photographs were taken and specific amplified bands at $310 \mathrm{bp}$ were looked for.

\section{Results}

A total of 310 blood samples were tested for $P$. falciparum infection. Of these, $160(51.6 \%)$ samples were positive for malarial parasite of which $63(39.4 \%)$ were positive for $P$. falciparum by Giemsa staining (one of these was diagnosed as mixed infection of $P$. falciparum and $P$. vivax.). Three cases which were missed earlier by Giemsa but were positive by PCR analysis were found to have very few asexual parasites with parasitaemia less than 100 parasites per microlitre of blood on extended (upto 600 fields) Giemsa examination. PCR analysis detected $61(38.1 \%)$ cases of $P$. falciparum. The case with mixed infection was positive by PCR analysis. However, two cases positive by Giemsa were not diagnosed by PCR analysis.

The PCR analysis for $P$. falciparum was found to be 96.8 $\%$ sensitive and $100 \%$ specific.

Giemsa staining required a total of 40-45 minutes, while PCR analysis needed 10-11 hours for each sample. Total handling time decreased for Giemsa and PCR analysis if the specimens were processed in larger batches. While Giemsa costs around only Rs. 2-3 per test, PCR analysis was many times costlier varying with the technique used for extraction of DNA and source of primer.

\section{Discussion}

Malaria remains a major tropical health problem and the resurgence of malaria, especially drug resistant cases of P.falciparum has renewed interest in developing preventive and accurate diagnostic methods for malaria.

Giemsa staining and demonstration of malarial parasites in peripheral blood through microscopic examination still

Table: A comparative evaluation of the tests done

\begin{tabular}{lcc}
\hline & Giemsa & PCR \\
\hline Sensitivity & & $96.8 \%$ \\
Specificity & & $100 \%$ \\
Time required & $40-45 \mathrm{~min}$ & $10-11 \mathrm{~h}$ \\
Cost/ test & Low & High \\
Expertise required & Moderate & High \\
Facilities required & Laboratory light & Special laboratory \\
& microscope & and equipment \\
\hline
\end{tabular}

remains the gold standard for definitive diagnosis of malaria. Blood collection for this technique is simple and can be performed with minimal training. Parasite species, stages and densities could be identified accurately as reported earlier. ${ }^{3}$ It was inexpensive and needed a limited amount of equipment. However, preparing a good quality of film needed practice and standardization for the dilution of Giemsa stain required, the staining time and preparation of the buffer with a proper $\mathrm{pH}$ to give adequate quality of stained films. The main disadvantage of this technique, as other studies have shown, is that it may give poor results in cases with low parasitaemia. ${ }^{3}$ It is difficult to interpret thick blood films when the parasitaemia is less than 500 per microlitre of blood. ${ }^{7}$ The PCR analysis for $P$. falciparum done in our study gave a sensitivity of $96.8 \%$. Other workers have reported a sensitivity varying from $79 \%{ }^{8}$ to more than $90 \% .^{9}$ The test was negative for two samples amongst a total of 63 P. falciparum cases in our study. Although PCR has been claimed to be theoretically capable of detecting the presence of a single parasite per microlitre of blood, this is rarely achieved. ${ }^{10}$ The negative results could be due to the presence of various inhibitory substances in blood like anticoagulants (heparin or EDTA) and intracellular substances (porphyrin ring of heme, which binds to the Taq 1 polymerase). Degradation of the target DNA due to extraction with repeated freezing and thawing or the presence of $P$. falciparum sequences that lack sequences recognized by primers or technical faults while DNA storage may give negative results. ${ }^{8}$ Specificity of PCR for $P$. falciparum in our study was $100 \%$. Other reports give a specificity of $69 \%{ }^{8}$ to more than $90 \% .^{9}$ One sample with mixed infection of $P$. vivax and $P$. falciparum was detected by PCR analysis in our study. Three cases which were missed earlier by Giemsa but were positive by PCR analysis were found to have very few asexual parasites with parasitaemia less than 100 parasites per microlitre of blood on extended (upto 600 fields) examination. Other studies have also reported the ability of PCR to diagnose mixed infections undetected by conventional microscopy. ${ }^{11}$ However, the technique is labour intensive and requires high level expertise and standardization. Time required was 10-11 hours for each sample, which is reduced if samples were processed in larger batches. However, it varies considerably with the technique used. PCR analysis is also more expensive requiring costly laboratory equipment instalment and maintenance.

The technique with new modifications offers increased sensitivity and specificity which may be significant in direct identification of drug resistant strains of parasites. It is also useful for other epidemiological studies, detection of mixed infections, low level parasitaemia and evaluation of chemotherapy and vaccine trials.

\section{References}

1. National Anti Malaria Programme, Ministry of Health and Family Welfare, Govt. of India. Malaria and its control in India, 
Country Scenario. Delhi: Directorate General of Health Services;2003.

2. Garcia LS, Bruckner DA. Malaria and Babesia spp. In: Diagnostic Medical Parasitology $2^{\text {nd }}$ edn. Garcia LS, Bruckner DA, Editors. Washington DC: American Society for Microbiology press; 1993. p. 113.

3. Craig MH, Sharp BL. Comparative evaluation of four techniques for the diagnosis of $P$. falciparum infections. Trans $R$ Soc Trop Med Hyg 1997;91:279-82.

4. Chayani N, Das B, Sur M, Bajoria S. Comparison of parasite lactate dehydrogenase based immunochromatographic antigen detection assay (OPTIMAL) with microscopy for detection of malaria parasites. Indian J Med Microbiol 2004;22:104-6.

5. Warhurst DC, Williams JE.Laboratory diagnosis of malaria. $J$ Clin Path 1996;49:533-8.

6. Viriyakosol S, Siripoon N, Petcharapirat C, Petcharapirat P, Jarra $\mathrm{W}$, Thaithong $\mathrm{S}$, et al. Genotyping of $P$. falciparum isolates by the polymerase chain reaction and potential uses in epidemiological studies. Bull W.H.O 1995;73:85-95.

7. Long GW, Jones TR, Rickman LS, Fries L, Egan J, Wellde B, et al. Acridine Orange diagnosis of $P$. falciparum: evaluation after experimental infection. Am J Trop Med Hyg 1994;51:6136.

8. Barker RH Jr, Banchongaksorn T, Courval JM Suwonkerd W, Rimwung tragoon K, Wirth DF. A simple method to detect $P$. falciparum directly from blood samples using the polymerase chain reaction. Am J Tr op Med Hyg 1992;46:416-26.

9. Makler MT, Palmer CJ, Ager AL.A review of practical techniques for the diagnosis of malaria. Ann Trop Med Parasitol 1998;92:419-33.

10. Topley and Wilson's Microbiology and Microbial Infections, 9th edn., New York: Oxford University Press; 1998.

11. Tirasophon W, Rajkulchai P, et al. A highly sensitive, rapid and simple polymerase chain reaction-based method to detect human malaria ( $P$. falciparum and $P$. vivax) in blood samples. Am J Trop Med Hyg 1993;87:647-8. 